\title{
TABLERO DE ALTAR DE ÉPOCA TARDOANTIGUA HALLADO EN BAZA (GRANADA). ¿EL PRIMER DOCUMENTO EPIGRÁFICO DEL OBISPO EUSEBIO?
}

\author{
POR \\ ALEJANDRO CABALLERO COBOS \\ Centro de Estudios de Arqueología Bastetana \\ HELENA GIMENO \\ Centro CIL II- Universidad de Alcalá de Henares \\ MANOLO RAMÍREZ AYAS \\ Centro de Estudios de Arqueología Bastetana \\ ISAAC SASTRE DE DIEGO \\ Instituto de Arqueología - Mérida. CSIC
}

\section{RESUMEN}

Presentamos el hallazgo en las proximidades de Baza de un fragmento de tablero de altar que conserva parte de una inscripción en el canto en la que se menciona a un obispo que, por la terminación del nombre, podría haberse tratado del obispo Eusebio, que acudió al IV Concilio de Toledo en el año 633. Esperamos que las futuras investigaciones en el yacimiento puedan confirmar esta hipótesis.

\section{SUMMARY}

This paper presents the discovery of an altar mensa fragment on the surroundings of Baza. The mentioned altar piece has remains of an inscription on its edge where a certain bishop is reffered to. Given the ending of his name, -bius, this bishop could have been Eusebius, who attended the IV Council of Toledo on 633. We are hoping that future research on the piece will confirm this hypothesis.

PALABRAS CLAVE: Baza, tablero de altar, época tardoantigua, obispo Eusebius?, epigrafía.

KEY WORDS: Baza, altar mensa, Late Antiquity, bishop Eusebius?, epigraphy.

\section{LA ANTIGUA BAZA. EL YACIMIENTO Y EL PROYECTO DE INVESTIGACIÓN}

En el año 2004 comenzó su andadura el Proyecto General de Investigación Iberismo y Romanización en el Área Nuclear Bastetana, aprobado por la Junta de Andalucía. Este proyecto se centra en la investigación del período ibérico y romano en la Hoya de Baza, a partir de la excavación del yacimiento de Cerro Cepero y la prospección del territorio circundante. Para dinamizar la gestión de los recursos los miembros del equipo de investigación fundaron en 2005 la Asociación de Estudios de Arqueología Bastetana (AEAB), entidad sin ánimo de lucro, con un fuerte compromiso con la investigación y difusión del patrimonio arqueológico de la comarca y que pretende encabezar la constitución del Centro de Estudios de Arqueología Bastetana en un futuro cercano.

A día de hoy se han realizado dos campañas de excavación arqueológica en el Cerro Cepero, una primera en 2004 de limpieza y documentación de estructuras de las antiguas excavaciones, y una segunda entre 2005 y 2006 que se centró en la excavación en extensión de la meseta superior del cerro. Estas actividades se acompañaron de dos campañas de prospección: una en 2004 en el campo de Jabalcón y otra en 2006 en el entorno de la ribera del río Baza. Además, la Asociación ha propiciado diversas actividades encaminadas a la difusión de estas investigaciones, como visitas teatralizadas al yacimiento con colegios de la comarca, cursos de formación, exposiciones sobre los resultados de la excavaciones, la fundación de una biblioteca sobre arqueología, y la creación de un sitio web (www.ceab.es).

La zona que nos ocupa se encuentra al Norte de la provincia de Granada, en lo que se conoce como Hoya de Baza o altiplanicie de Baza, formando parte del rosario de depresiones que ocupan el surco intrabético, rellenadas por materiales de origen sedimentario y de edad neógeno-cuaternaria. Su origen es tectónico, formada en el Mioceno Superior, tras la fase de orogenia alpina de las Cordilleras Béticas. Se comporta como una cuenca de sedimentación de materiales de origen marino y continental con un relleno compuesto por materiales detríticos procedentes de los relieves circundantes. Presenta un relieve tabular, aparentemente llano, que sin embar- 


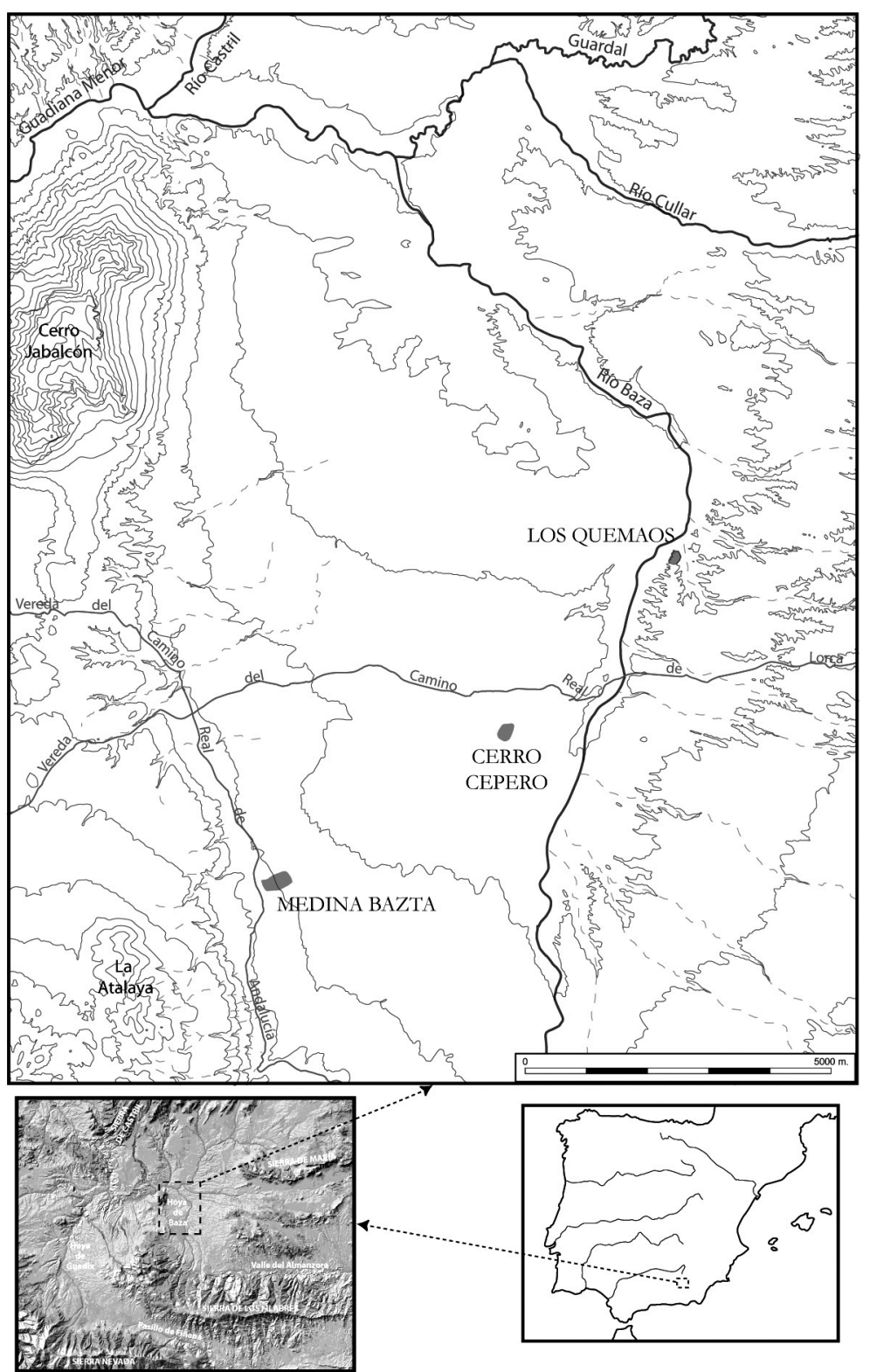

Figura 1. Localización de los yacimientos.

go está cortado por una densa red hidrográfica de estructura dendrítica, cuyo eje principal lo compone el río Guadiana Menor, el principal afluente de la cuenca alta del Guadalquivir.

El rasgo geográfico que más caracteriza este espacio es el de ser una altiplanicie con una elevada altura sobre el nivel del mar, en torno a los 1.000 metros en sus bordes, lo que le confiere una acusada continentalidad, rodeado por las sierras de Castril, María, Filabres y la propia sierra de Baza. Se localizan tres accesos o luga- res de paso naturales a la Hoya; uno de ellos viene representado por el valle del río Almanzora entre Sierra de Filabres y Sierra de María, funcionando como acceso natural hacia la costa almeriense; una segunda vía de acceso sería la localizada al noroeste de la Hoya, definida por la cabecera del río Guadiana Menor, que daría acceso hacia la Hoya de Guadix y la Alta Andalucía, y una tercera se localiza entre las sierras de Castril y de María, a través de las cuencas de los ríos Huéscar y Galera hacia la zona de Caravaca y Murcia. 


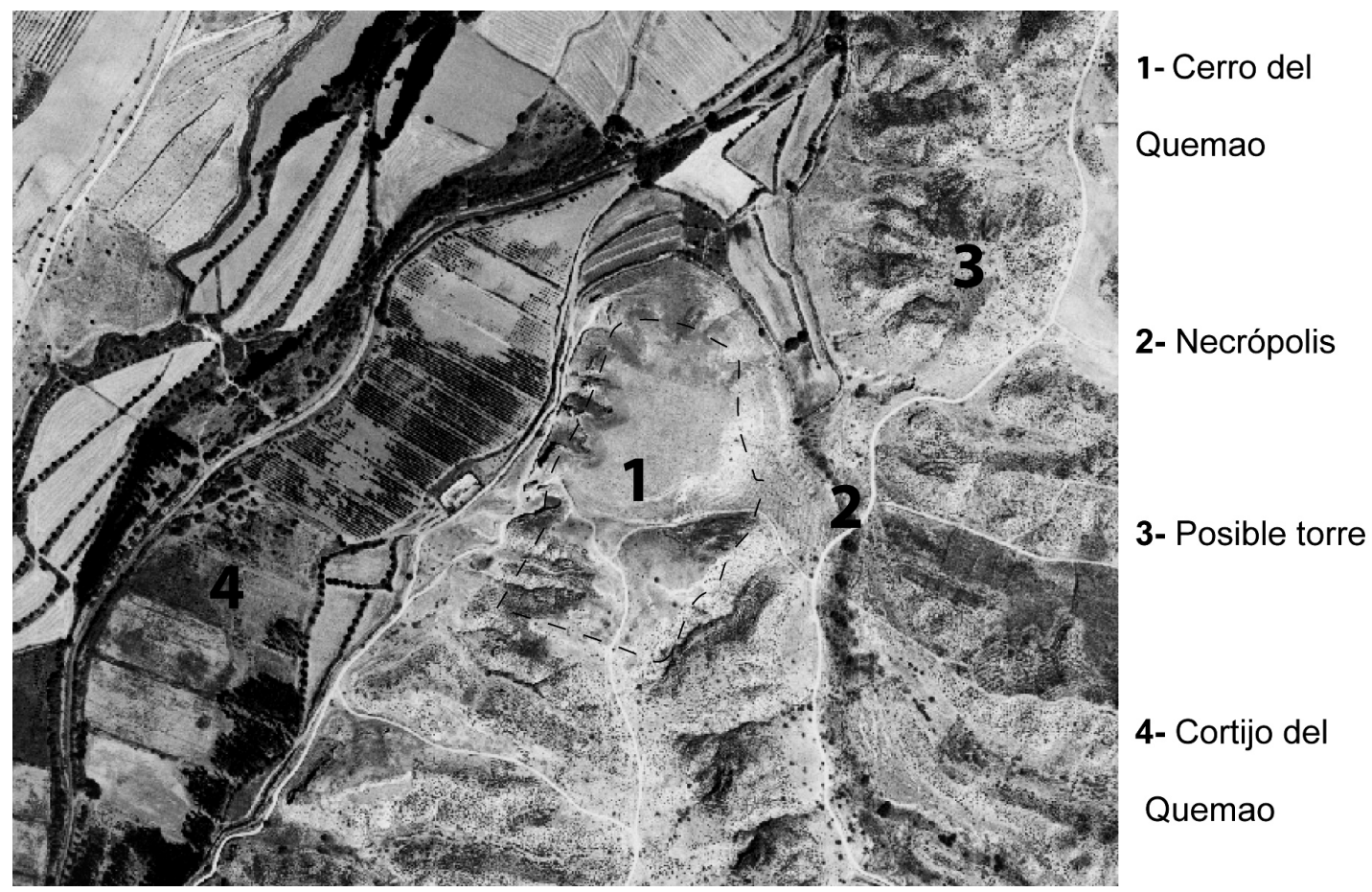

Figura 2. Cerro de los Quemaos y su entorno.

En Cerro Cepero se localiza el oppidum de la necrópolis ibérica del Cerro del Santuario, lugar de hallazgo de la célebre Dama de Baza (PRESEDO, 1982). Con la romanización el yacimiento experimentó una gran transformación, que se traduce en la presencia de edificios termales y cultuales (MARIN, 1992), que nos permiten inferir la hipótesis de que este lugar es el asiento de la antigua Basti ${ }^{1}$. La última campaña de excavación en Cerro Cepero ha permitido conocer con amplitud la última fase de ocupación del hábitat, que se concreta en el reaprovechamiento de una serie de edificios de cronología altoimperial en época visigoda tardía (siglo VI-VII), acabando la ocupación del mismo como muy tarde en el siglo VIII.

En el marco de la prospección de la ribera de Baza, a finales de mayo del 2006, documentamos un yacimiento que denominamos Cerro de los Quemaos, donde localizamos un fragmento de mármol con una inscripción latina en uno de sus cantos. Este elemento es el objeto de esta noticia.

El Cerro de los Quemaos se sitúa en la margen derecha del río Baza, en el borde oriental de la Hoya de Baza,

\footnotetext{
1 MARIN, N., 1992: "Baza y su comarca durante la época romana.", Granada. PRESEDO, F., 1982: "La necrópolis de Baza.", Excavaciones Arqueológicas en España, 119, Madrid.
}

dentro de un contexto geomorfológico de bad-lands. Se trata de una meseta formada por la confluencia del río Baza con el barranco del Espartal, con desniveles de hasta $40 \mathrm{~m}$ con respecto al valle, y cuyo mejor acceso se realiza desde el sur. El yacimiento presenta una extensión aproximada de 4.5 ha y se encuentra fuertemente alterado, siendo posible apreciar en superficie gran cantidad de estructuras como muros, silos y estructuras hidráulicas. El material arqueológico recogido en superficie evidencia una fuerte ocupación andalusí que como mínimo comienza en el siglo X y se prolonga hasta el XII, aunque también hemos documentado algunas cerámicas romanas muy fragmentadas, que permiten valorar la existencia de una fase de ocupación romana, que comenzaría como muy temprano en el siglo I d.C. y que se continuaría durante los siglos IV y V, siendo muy difícil precisar la continuidad de esa ocupación hasta el período andalusí. En las estructuras murarias conservadas se observa una técnica basada en la combinación del yeso con cantos de río, sin presencia de ladrillo ni sillería.

En torno a esta meseta hemos documentado algunos yacimientos que merecen la pena ser reseñados. En primer lugar, al Este, en el fondo del barranco del Espartal se conoce un área que identificamos como una necrópolis de inhumación. En segundo lugar, también hacia el Este, sobre un cerro amesetado, se localiza una estructura cuadrangular muy arrasada, formada por cantos de río 
dispuestos en espina de pez, con escasísimo material cerámico, tanto romano como medieval, que podría tratarse de una torre cuadrada con partición interna. En tercer lugar al noreste, en la margen izquierda del río Baza, cercano al cortijo de los Quemados, sobre un relieve de terrazas, existe un área con material cerámico altoimperial y bajoimperial, en el que también se aprecian algunas inhumaciones cubiertas con lajas de caliza, de cronología altomedieval.

\section{EL ALTAR DE BAZA Y SU CONTEXTO EN LOS ALTARES EN EL SIGLO VII}

Como acabamos de señalar, el fragmento fue hallado en la última campaña de prospecciones llevada a cabo recientemente en "Cerro del Quemao", en su parte alta. Se encontró en una zona superficial con gran cantidad de lajas de piedra. Se trata de una placa de mármol blanco de Macael de 0,66 x 0,27 x 0,065-0,03 m de grosor, rota por detrás y por ambos lados, en cuyo plano superior presenta un tipo de decoración característica de los tableros de altar de época tardoantigua; no encaja mal con este tipo de monumento el texto que está grabado en el canto.

El marco del plano superior está compuesto por una serie de molduras en bajorrelieve que miden en total 13 $\mathrm{cm}$ de ancho, presentando la siguiente disposición: dos pequeñas molduras biseladas de $1,5 \mathrm{~cm}$ de anchura a modo de marco interno que dan paso a un acanaladura recta más ancha y que termina con la moldura lisa del borde, de $5 \mathrm{~cm}$ de anchura. En el resto de la superficie del plano superior aparecen marcas de haber sido picada, aunque ignoramos a qué momento pueden pertenecer. En cuanto al plano inferior, conserva las marcas del serrado en el sentido de las molduras del marco del plano superior; en la zona del borde aparece una muesca, un retranqueo de $1 \mathrm{~cm}$ a modo de chaflán en casi todo el largo de la pieza, posiblemente debido al propio corte de la pieza mediante sierra. El grosor de la placa disminuye desde el borde hacia el centro (de 6,5 a $3 \mathrm{~cm}$ ).

La inscripción que recorre el canto -en general bien conservada, pero con concreciones en algunas partes mide $5 \mathrm{~cm}$ de alto y ocupa todo el largo conservado 66 $\mathrm{cm}$. Las letras, capitales, de 3-4 cm, de tamaño irregular, muy separadas y de factura tosca, no respetan la alineación del renglón sino que tienden a salirse de la caja por arriba y llaman la atención por la profundidad tan distinta que presentan en la incisión, mayor en el lado izquierdo. La interpunción es una hedera. El texto dice:

\section{[- - -]BIVS AEPISCOPVS • OMNIVM [- - - ]}

Las letras presentan algunas particularidades curiosas como el travesaño angular de la A algo curvado, la primera $\mathrm{V}$ que no cierra en el vértice o el trazo recto de la $\mathrm{C}$ mucho más frecuente en inscripciones postvisigodas y, sobre todo, llama la atención su mala ejecución tanto en las formas como en la incisión. También es relevante la utilización del dígrafo $\mathrm{AE}$, una hipercorrección ortográfica bien constatada en época antigua ${ }^{2}$ y muy frecuente en inscripciones a partir del medioevo. No sabemos cuánto falta por la izquierda pero sí que hay que completar el nombre del obispo y que, por la derecha, es muy difícil aventurar ninguna restitución y ni siquiera podemos calcular lo que falta. Se puede pensar que al ser el obispo el personaje que realiza la acción o éste está al comienzo del texto (lo más probable) o, por el contrario, lo cierra. En cualquier caso, la interpunción marca una separación para destacar el nombre y el cargo del obispo. Por el tipo de letra, y sin contexto arqueológico, tampoco es posible aventurar una fecha segura, máxime cuando las letras son de tan mala factura. Sería, por tanto, necesario excavar con urgencia en el yacimiento para ver la cronología que aportan el resto de los materiales, eso considerando que la pieza se encuentre en el ámbito de su contexto original.

Terreno más firme pisamos al analizar la pieza desde un punto de vista material, ya que encaja con los tableros de altar cuya ejecución decorativa es característica de los talleres de la segunda mitad del siglo VI y del siglo VII, algo que se aprecia en la talla de la doble moldura biselada que recorre la pieza junto al borde. Este tipo de marco está presente en otros tableros de altar hispanos de este periodo, aunque cada uno con sus propias variantes ${ }^{3}$. Un ejemplo similar de doble moldura biselada interior, aquí sin acanaladura intermedia entre aquellas y el borde, lo encontramos en la esquina de tablero de Valdecebadar (Olivenza), conservado en el Museo de Badajoz $^{4}$. Pero no es algo exclusivo de la Península Ibérica, existen composiciones parecidas en las que se juega con el número y forma de las molduras en otras áreas del Mediterráneo ${ }^{5}$. Lo que tampoco podemos resolver es si se trata de una pieza anterior -romana-

\footnotetext{
2 Concretamente para la palabra (a)episcopus véase bajo este vocablo en el índice VII de Diehl, ILCV, p. 349, apartado Aa.

No deja de ser una herencia de los marcos moldurados de las placas epigráficas romanas.

4 Agradecemos a Guillermo Kurtz, director del Museo de Badajoz, y a Coronada Domínguez, conservadora del mismo, todas las facilidades que nos han dado para consultar esta pieza, también estudiada por Tilo Ulbert, quien publicará próximamente un trabajo sobre el yacimiento de Valdecebadar y a quien también agradecemos los datos que nos ha aportado sobre los restos con carácter litúrgico procedentes de este edificio.

5 Una revisión actualizada en VV. AA., 2005: The altar from 4th to the 15 th century, Hortus Artium Medievalium. Journal of the International Research Center for Late Antiquity and Middle Ages, vol. 11, Zagreb-Motovum.
} 

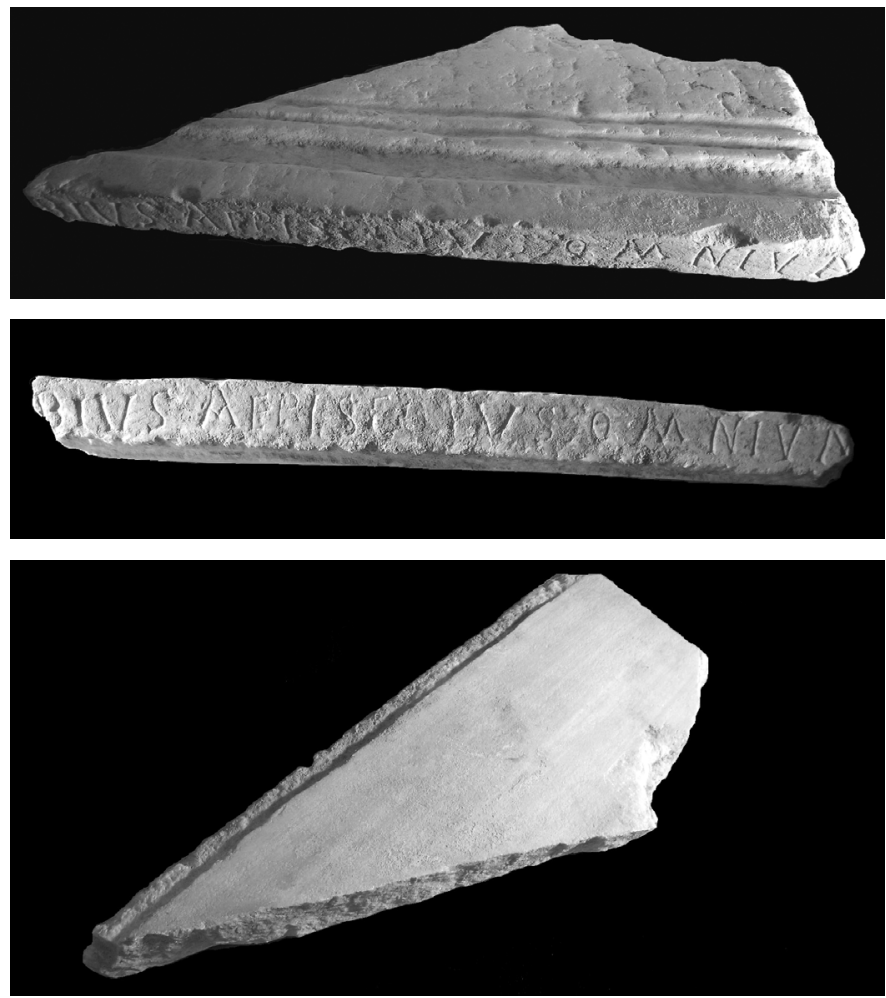

C

Figura 3. Tablero de altar de Cerro de los Quemaos, Baza. a: plano superior; b: canto; c: plano inferior.

reutilizada ${ }^{6}$, o si está específicamente cortada y labrada para su función tardoantigua; tampoco la procedencia

\footnotetext{
${ }^{6}$ La reutilización de piezas romanas, especialmente aras y pedestales, como partes de altares cristianos es común en la Antigüedad Tardía. Un ejemplo muy ilustrativo lo encontramos en el altar de Ginés, actualmente en el Museo Arqueológico de Sevi1la. Se trata de un ara romana transformada en ara cristiana, no sólo por la refacción del foculus romano en loculus cristiano, sino por la inscripción realizada picando la anterior inscripción pagana. Tras el crismón aparece la dedicación por parte de un obispo. Ver: J. González: Corpus Inscripciones Latinas de Andalucía, vol. II, tomo II, no 591, fig. 340; J. Beltrán Fortes, 1991: "Altares visigodos. Reutilizaciones paganas (I)", en S. Ordoñez, P. Sáez (coord.): Homenaje al profesor Presedo, 785-810. El gusto por la constancia escrita en los altares se concentra más en el Sur, en la actual Andalucía. Muy cerca de Baza, en Guadix (M. Pastor Muñoz, Corpus de inscripciones latinas de Andalucía. IV: Granada, Sevilla 2002, n 137) la antigua Acci, se encontró un pedestal de estatua dedicada a Magnia Urbica entre el 283 y el 285, que se reutilizó como altar en el siglo VII y donde se enumeran gran cantidad de reliquias de algunos de los santos y mártires más famosos de la Hispania tardorromana y visigoda como son Santa Eulalia, San Vicente, las Santas Justa y Rufina, etc. La pieza actualmente está perdida y no se conoce imagen de ella. También son famosas las consagraciones del obispo Pimenio en
} la zona de Cádiz durante la segunda mitad del siglo VII. del mármol de Macael resuelve el problema, ya que Macael es una importante cantera que abastece a una amplia zona de la Península Ibérica desde época romana y, por tanto, su mármol sigue utilizándose en la Antigüedad Tardía.

Como la inscripción está incompleta, pues además de parte del nombre del obispo falta detrás de omnium al menos el sustantivo del que era complemento y como mínimo el objeto de la dedicación y el mismo verbo que indicaba la acción que se realizaba, la longitud del tablero debió ser mayor. Es imposible siquiera intentar una aproximación pues no sabemos si la inscripción ocupó solo el frente, o todos, o tres de los lados como ocurre en el tablero perdido del altar de Salpensa ${ }^{7}$ cuya inscripción discurría, a modo de marco, por tres de sus lados aunque en este caso no estaba inscrita en el canto sino en el plano superior. Ahora bien, en la Península Ibérica es más usual encontrar los textos asociados a los altares en los soportes de las aras que en las mensae, por lo que el tablero de Baza reviste particular interés, aunque los

J. Beltrán Fortes, cit. (n. 9), 788, nota 20, fig. 1. Además del tablero de Salpensa tendríamos otro posible paralelo en el altar de Santa María del Naranco (Oviedo), aunque es un siglo posterior. 
escasos restos de la inscripción y el estado fragmentario de la pieza nos impiden determinar a qué modelo de altar perteneció. Si la inscripción ocupó solo el frente, con lo que el tablero habría alcanzado fácilmente más de un metro de longitud, magnitud propia de los tableros de altar de soporte múltiple, el altar habría tenido cuatro o cinco pies; pero si recorría los cuatro cantos, o sólo tres la mesa habría sido menor y habría más dudas para asignarle un soporte único o múltiple o incluso de tipo bloque. En la parte que falta de la inscripción y, teniendo en cuenta el soporte, esperaríamos un texto relativo o bien a la deposición de las reliquias de los santos en el altar o a su memoria, o a la consagración del mismo, o, en menor medida, a su construcción. Menos probable sería que aludiera a la dedicación o consagración de la iglesia, o a la construcción/reparación de ella o de alguna parte de ella. Tampoco sería imposible que omnium formara parte de un texto poético ${ }^{8}$. De todas estas posibilidades y en relación con su soporte, preferiríamos la primera, es decir, que se refiriera a la deposición de las reliquias o a la consagración del altar, un texto del que nos pueden dar una idea las líneas 6 y 7 de la inscripción del antistes Honoratus ${ }^{9}$, que conmemora la fundación y dedicación de un templo a los mártires cordobeses y la consagración de un ara. Este texto, que no sólo se refiere al altar, está grabado en una placa que sin duda estaría incrustada en alguna parte relevante de la iglesia y, por su longitud, $124 \mathrm{~cm}$, no es descabellado pensar que se tratara de una entrada.

En cuanto al nombre del obispo, a pesar de lo poco que de él se ha conservado y de que existen más nombres personales terminados en -bius ${ }^{10}$, es muy tentador relacionarlo con el "Eusebius ecclesiae Bastitanae episcopus ${ }^{11}$ que firma en decimoséptimo lugar en el IV Concilio de Toledo (633) y que vuelve a firmar tres años después en el V Concilio de Toledo, esta vez ya en sép-

8 Un texto de este tipo aparece en un altar de Rubí (G. Fabre - M. Mayer, I. Rodà, Inscriptions Romaines de Catalogne I, Paris 1984, $n^{\circ}$ 64) cuyos editores más recientes interpretan como buena muestra de sermo humilis por su contenido: + Felici misero penarum pondera pelle $\|+($ Christe $)$ D(ominu $)$, per cuncta pius qui s(ae)c(u)la regnas. Hic s(an)c(tu)s semper sedito, hic abitator adesto $\|+$ Felici misero tota tu tristia tolle.".

9 ... antistes Honoratus ... hic aram in medio sacrans altare reconditfratres sanctos retinet quos Cordoba passos aedem deinde trium sanctorum iure dicavit (J. González, Corpus de Inscripciones Latinas de Andalucía. II, Sevilla. II. La Vega (Itálica) no 606).

${ }^{10}$ Cf. H. Solin - O. Salomies, Repertorium nominum gentilium et cognominum Latinorum, Hildesheim - Zürich- New York, 1988, 223 donde puede verse una nómina bastante abundante de nombres terminados en -bius además de Eusebius.

11 J. Vives, 1963: Concilios visigóticos e hispano-romanos, vol. 1,222 . timo lugar, y en el VI (638). Si esta identificación fuera cierta ${ }^{12}$, el tablero constituiría el primer testimonio arqueológico conservado de la sede episcopal visigoda bastetana y de su obispo Eusebio; y adquiere así una especial relevancia al ofrecer nuevos datos para el conocimiento de la ciudad bastetana y del cristianismo del Sureste peninsular en época visigoda.

Sin embargo, siguen siendo muchas las cuestiones abiertas que todavía existen sobre el territorio y la ciudad de Baza durante la Antigüedad Tardía. Los datos presentados, aún importantes, no permiten una conclusión cerrada acerca de la cuestión de la localización de la sede episcopal de Basti, puesto que la ubicación original del tablero de altar no es segura. Éste pudo ser reutilizado en época islámica como elemento de construcción, o incluso haber sido trasladado a una nueva iglesia por obispos posteriores en época medieval. Tampoco es posible afirmar que la pieza perteneciera a la propia iglesia episcopal, puesto que también podría corresponder a una parroquia rural del entorno de Cerro de los Quemaos consagrada por el obispo bastetano.

La cuestión de fondo es el traslado del asentamiento visigodo de Cerro Cepero a Medina Bazta, la actual Baza, en el intervalo de tiempo entre el siglo VIII y X. Creemos que la supervivencia del topónimo Basti-Bazta indica que hubo un factor político que permitió el traslado de dicha sede, que pensamos fue el obispado. En cualquier caso, lo que sí permite este hallazgo es asociar claramente el obispado tardoantiguo de Baza a un espacio geográfico específico como es el centro de la Hoya de dicho nombre.

Recibido el 05-09-06 Aceptado el 10-09-06

\footnotetext{
${ }^{12}$ A favor de esta propuesta está el hecho de que, de los nombres conservados a través de las actas conciliares de obispos bastetanos, sólo se conoce un caso de nombre acabado en -bius, el obispo Eusebio del IV, V y VI Concilio de Toledo.
} 